Journal of Universal Mathematics

VOL.2 No.1 PP.32-35 (2019)

ISSN-2618-5660

\title{
EFFECTIVE MASS KLEIN-GORDON EQUATION WITH POSITION DEPENDENT MAGNETIC FIELD
}

\author{
O. MIZRAK, O. AYDOGDU, AND K. SOGUT
}

\begin{abstract}
Recently, exact solutions of the classical and relativistic wave equations for the existance of an external potential have been widely studied in view of the position-dependent mass formalism. Such efforts have important applications in technology, especially in material science such as electronic properties of the semi-conductors and quantum dots. In the present study, we aim to obtain exact solutions of the Klein-Gordon equation in the presence of an exponential magnetic field via effective mass formalism. Energy eigenvalues are derived by using wave functions. The studied magnetic field and effective mass have the form $\vec{B}=B_{0} e^{-\kappa x} \hat{k}$ and $m(x)=\left(m_{0}+m_{1} e^{-\kappa x}\right)$.
\end{abstract}

\section{INTRODUCTION}

The investigation of a non-relativistic or relativistic quantum mechanical system under the sway of the external fields has got great attention over the past decades. For this purpose Schrödinger, Klein-Gordon and Dirac wave equations are solved in the existance of the external fields [1]-[5].

Of these equations, the Schrödinger equation is the most natural way of starting point of the quantum mechanical description of subatomic particles. It is the fundamental equation for the description of the non-relativistic phenomena.

The Klein-Godon equation is the precursor equation to start the relativistic phenomena of subatomic particles. But, similar to Schrödinger equation, it does not address the spin.

The Dirac equation is the primary equation that includes both relativistic effects and spin. It's theoretical predictions agree very well with experimental results with very high sensitivity. In nuclear, high energy and condensed matter physics, etc., the Klein-Gordon and Dirac equations are solved exactly in order to evaluate the relativistic effects. In many cases of these equations, the solutions are obtained for constant or time-dependent mass. To our knowledge, presently the effective mass solutions are less obtained for these equations.

Solving the Schrödinger equation for the position-dependent mass is rather more common and so far it has been solved for many different cases of scalar and vector potentials. In these problems, in order to solve the problem analytically the

Date: Review January 2, 2018, accepted January 22, 2019.

2000 Mathematics Subject Classification. Primary xxx, yyyy; Secondary xxxx, yyyy.

Key words and phrases. Klein-Gordon equations, external fields, exact solutions. 
position-dependent mass form has been taken similar to the form of potential [6][11]. Such efforts have been presented also for the Klein-Gordon equation. Approximate and exact analytic solutions have been obtained for several potential types with position-dependent mass [12]-[16].

In the present study, we aim to obtain exact analytic solutions of the KleinGordon equation for a position-dependent exponentially decaying magnetic field with a position-dependent mass in the similar mathematical form. Eigenfunctions and energy spectrum are obtained for the considered case. Such kind of decaying magnetic field has been studied for the Dirac electron in graphene and a single 2D electron Pauli equation.

The paper is organized as follows: in section 2 we obtain the exact solutions of the Klein-Gordon equation, in section 3 the dependence of the energy spectrum on the varying mass and magnetic field is investigated. Section 4 is dedicated to discussion.

\section{Exact Solution of the Problem}

The Klein-Gordon equation for relativistic spinless particles in the presence of external fields with mass $m$ is given by [2]:

$$
\left[(\vec{P}-e \vec{A})^{2}+m^{2}(x)\right] \varphi=\left(P_{0}-e A_{0}\right)^{2} \varphi
$$

where $A_{\mu}$ is the electromagnetic four-vector potential, $e$ is the charge of the particle, $P_{\mu}$ is four-vector momentum and $m(x)$ is the position-dependent mass of particle.

If we work for the vector potential with the gauge

$$
A_{\mu}=\left(0,0, A_{y}, 0\right)=A_{y}(x)=-\frac{1}{\kappa} B_{0} e^{-\kappa x}
$$

the magnetic field will be as $\vec{B}=B_{0} e^{-\kappa x} \hat{k}$. For the mass $m(x)=\left(m_{0}+m_{1} e^{-\kappa x}\right)$, the Klein-Gordon equation reads

$\left[\frac{d^{2}}{d x^{2}}-\left(\frac{e^{2} B_{0}^{2}}{\kappa^{2}}+m_{1}^{2}\right) e^{-2 \kappa x}-\left(\frac{2 e k_{y} B_{0}}{\kappa}+2 m_{0} m_{1}\right) e^{-\kappa x}+\varepsilon^{2}-\left(m_{0}^{2}+k_{y}^{2}+k_{z}^{2}\right)\right] \psi(x)=0$ where the ansatz for the wave function is $\varphi=e^{i\left(y k_{y}+z k_{z}-\varepsilon t\right)} \psi(x)$.

By making change of variable $u=e^{-\kappa x}$ and defining $\psi(u)=u^{\frac{-1}{2}} F(u)$, Eq. (2.3) returns

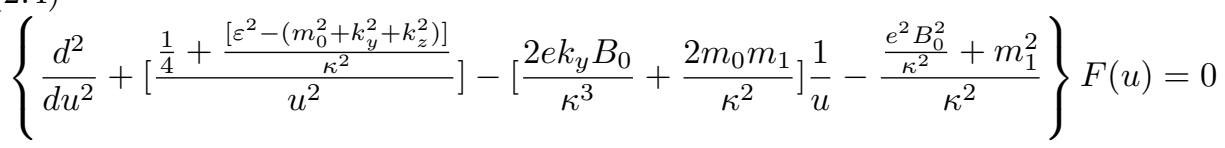

Defining a new variable, $\rho=2\left(\frac{\sqrt{\frac{e^{2} B_{0}^{2}}{\kappa^{2}}+m_{1}^{2}}}{\kappa}\right) u$, this equation reduces into the following form:

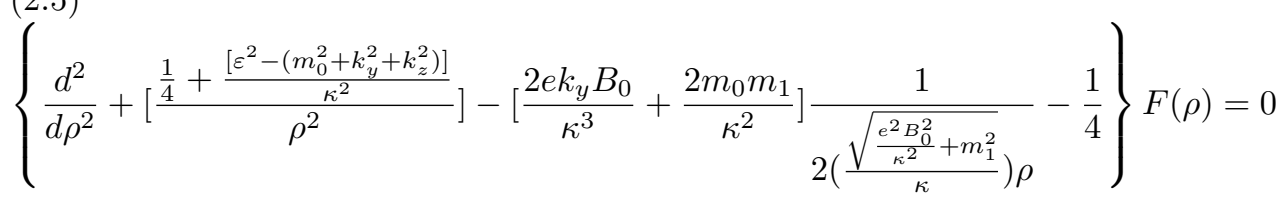


Definition 2.1. The Whittaker equation is given by [17]

$$
\frac{d^{2} F(\rho)}{d \rho^{2}}+\left[-\frac{1}{4}+\frac{\lambda}{\rho}+\frac{\frac{1}{4}-\mu^{2}}{\rho^{2}}\right] F(\rho)=0
$$

The general solution of this differential equation is given in terms of the Whittaker functions by

$$
F(\rho)=N_{1} M_{\lambda, \mu}(\rho)+N_{2} W_{\lambda, \mu}(\rho)
$$

where $N_{1}$ and $N_{2}$ are arbitrary constants.

Then, solutions of equation (2.5) are given by equation (2.7) with $\lambda$ and $\mu$ are defined as follows: $\mu=\frac{i}{\kappa} \sqrt{\varepsilon^{2}-\left(m_{0}^{2}+k_{y}^{2}+k_{z}^{2}\right)}$ and $\lambda=\frac{\left(\frac{2 e k_{y} B_{0}}{\kappa}+m_{0} m_{1}\right)}{\sqrt{e^{2} B_{0}^{2}+m_{1}^{2} \kappa^{2}}}$.

For the requirement that the Whittaker functions must be bounded for the variable we have the expression [17]

$$
\frac{1}{2}+\mu-\lambda=-n
$$

where $n$ is a positive integer. By using the explicit values of $\mu$ and $\lambda$ in this expression, we obtain energy spectrum equation as

$\varepsilon^{2}=\left(m_{0}^{2}+k_{y}^{2}+k_{z}^{2}\right)+\kappa^{2}(2 n+1) \frac{\left[\frac{e k_{y} B_{0}}{\kappa}+m_{0} m_{1}\right]}{\sqrt{e^{2} B_{0}^{2}+m_{1}^{2} \kappa^{2}}}-\left[(\kappa n+\kappa)^{2}+\frac{\left(\frac{e k_{y} B_{0}}{\kappa}+m_{0} m_{1}\right)^{2}}{e^{2} B_{0}^{2}+m_{1}^{2} \kappa^{2}}\right]$

\section{Effect of Varying Mass and Field on Energy Spectrum}

3.1. Case I $\left(B_{0}=0\right)$.

$$
\varepsilon^{2}=\left(m_{0}^{2}+k_{y}^{2}+k_{z}^{2}\right)+\kappa(2 n+1) m_{0}-\left[(\kappa n+\kappa)^{2}+\frac{m_{0}^{2}}{\kappa^{2}}\right]
$$

As it is seen the result does not depend on the $m_{1}$ factor of varying mass explicitly, but effects coming from the varying mass manifest in the spectrum with $\kappa$ term.

3.2. Case II $\left(m_{1}=0\right)$. For this case the energy spectrum reads

$$
\varepsilon^{2}=\left(m_{0}^{2}+k_{y}^{2}+k_{z}^{2}\right)+\kappa(2 n+1) k_{y}-\left[(\kappa n+\kappa)^{2}+\frac{k_{y}^{2}}{\kappa^{2}}\right]
$$

We see an intresting point here compared to the Case $I$; in the second and third terms of energy spectrum the constant mass exchanged with $k_{y}$.

\section{Conclusion}

In the present study, we obtain exact solutions of the effective mass Klein-Gordon equation with exponentially decaying magnetic field. Energy spectrum of the relativistic particles is derived by using the wave functions. There are fewer studies in the literature with variable masses compared to constant mass. Besides, our problem is important since the magnetic field we considered here occurs inside the London penetration depth of a superconductor of first kind [18] for a better realization for materials such as graphene. 


\section{REFERENCES}

[1] P. J. Redmond, Solution of the Klein-Gordon and Dirac equations for a particle with a plane electromagnetic wave and a parallel magnetic field, Journal of Mathematical Physics, vol. 6, pp. 11631169, (1965).

[2] L. Lam, Motion in electric and magnetic fields. I. Klein-Gordon particles, Journal of Mathematical Physics, vol. 12, no.2, pp. 299303, (1971).

[3] G. Ivanovski, D. Jakimovski, and V. Sopova, Energy levels of a charged particle in a homogeneous electric field orthogonal to a piecewise homogeneous magnetic field, Physics Letters A, vol. 183, no. 1, pp. 2428, (1993).

[4] V. M. Villalba and R. Pino, Energy spectrum of a relativistic two-dimensional hydrogen-like atom in a constant magnetic field of arbitrary strength, Physica E: Low-Dimensional Systems and Nanostructures, vol. 10, no. 4, pp. 561568, (2001).

[5] K. Sogut, H. Yanar, A. Havare, "Production of Dirac Particles in External Electromagnetic Fields", Acta Physica Polonica B, No 9, Vol. 48, (2017).

[6] L. Dekar, L. Chetouani and T. F. Hamann,"Wave function for smooth potential and mass step", Physical Review A 59, 107, (1999).

[7] A. S. Dutra and C. A. S. Almeida, "Exact solvability of potentials with spatially dependent effective masses", Physics Letters A 275, 25, (2000).

[8] R. Koc, M. Koca and E. Korcuk, "A new class of quasi-exactly solvable potentials with a position-dependent mass", Journal Physics A, 35, L527, (2002).

[9] B. Gonul, O. Ozer, B. Gonul B and F. Uzgun, "Exact Solutions of Effective-Mass Schrödinger Equations" Modern Physics Letters A, 17, 2453, (2002).

[10] C. Tezcan and R. Sever, "Exact Solutions of the Schrödinger Equation with Positiondependent Effective Mass via General Point Canonical Transformation", Journal of Mathematical Chemistry, 42, 387,(2007).

[11] R. Sever and C. Tezcan, "Exact Solution of the Schrödinger Equation for the Modified Kratzer's Molecular Potential With Position-Dependent Mass" International Journal of Modern Physics E, 17, 1327, (2008).

[12] A. D. Alhaidari, "Solution of the Dirac equation with position-dependent mass in the Coulomb field", Physics Letters A, 322, 12, (2004).

[13] O. Mustafa and S. H. Mazharimousavi, "First-Order Intertwining Operators with Position Dependent Mass and -Weak-Pseudo-Hermiticity Generators", International Journal of Theoretical Physics, 47, 446, (2008).

[14] C. S. Jia and A. S. Dutra, "Extension of PT-symmetric quantum mechanics to the Dirac theory with position-dependent mass", Annals of Physics, 323, 566, (2008).

[15] A. Arda, R. Sever, C. Tezcan, "Analytical Solutions to the KleinGordon Equation with Position-Dependent Mass for q-Parameter Pöschl-Teller Potential", Chinese Physics Letters Vol. 27, No. 1, 010306, (2010).

[16] O. Aydogdu, A. Arda and R. Sever, "Scattering of a spinless particle by an asymmetric Hulthn potential within the effective mass formalism", Journal of Mathematical Physics 53, 102111, (2012).

[17] M. Abramowitz and I. A. Stegun, Handbook of Mathematical Functions, Dover, New York, NY,USA, (1970).

[18] K. Handrich, "Quantum mechanical magnetic-field-gradient drift velocity:An analytically solvable model", Physical Review B, 72, 161308(R), (2005).

(1) Mersin university, mathematics department, 33343, mersin, TURKey

E-mail address: mizrak@mersin.edu.tr

(2) MERSIN UNIVERSITY, PHYSICS DEPARTMENT, 33343, MERSIN, TURKEY

E-mail address: oktaydogdu@gmail.com

(3) MERSin UniVERsity, PHYSiCs DEPARTMENT, 33343, MERSin, TURKEY

E-mail address: kenansogut@gmail.com 